\title{
Selected issues of the DGPPN Congress in 2011
}

\author{
Andrea Schmitt • Oliver Gruber • Peter Falkai
}

Published online: 4 October 2012

(c) Springer-Verlag Berlin Heidelberg 2012

This issue deals with a wide and multifocal thematic range of interesting scientific contents presented at the DGPPN Congress in November 2011. Starting with the DGPPN research project on mental healthcare utilization in Germany, Gaebel et al. [1] provide insight into increased utilization of psychiatric and psychosomatic disciplines in both in- and outpatient treatment compared to general medical facilities for 1.4 million patients with depression in Germany from 2005 to 2007. Within this group, specialists for psychosomatic medicine and psychological psychotherapists performed care of mild and moderate depression, whereas psychiatrists were assigned to treat the severe cases. Härter et al. [2] describe the newly implemented Hamburg Network for Mental Health as trans-sectoral cooperation including innovative measures to improve treatment for mental disorders, which derive from highquality research in psychosis, depression, somatoform and functional syndromes, anorexia, bulimia and addiction illnesses. The aspect of stress, burnout and job dissatisfaction in mental health workers is covered by Rössler [3]. Beside well-known stressors such as extensive workload or long night shifts, he identifies stigma of the profession including demanding relationships with patients and difficult interactions with other health professionals, threats from violent patients, suicide plus a lack of positive feedback as specific

\footnotetext{
A. Schmitt · P. Falkai ( $₫)$

Department of Psychiatry and Psychotherapy,

Ludwig-Maximilians-University Munich,

Nußbaumstr. 7, 80336 Munich, Germany

e-mail: Peter.Falkai@med.uni-muenchen.de

O. Gruber

Department of Psychiatry and Psychotherapy, University of Göttingen, von-Siebold-Str. 5, 37075 Göttingen, Germany
}

challenges, possibly leading even to post-traumatic stress symptoms and burnout.

Genius et al. [4] reviews the performance of current CSF biomarkers and their interpretation in differential and early diagnosis of Alzheimer's disease, which is important in light of increasing prevalence of the disease. New prospective markers including the role and limitation of genotyping are discussed here. In schizophrenia, to date, biomarkers are missing, but the group of Bahn et al. [5] developed the first validated blood test of schizophrenia, including proteins of metabolic, immune and hormonal imbalances. Further research of biomarkers for diagnostic applications, extending to functional analysis of blood cells and prediction of drug response may represent thrilling research avenues in the future. On the other hand, prevention of psychosis symptoms is regarded as a promising strategy to improve unfavorable outcome of the disease. Ruhrmann et al. [6] report on error probability and costbenefit relationship in predictive approaches, identifying risk criteria in psychosis.

Auditory verbal hallucinations are known as one of the core symptoms of psychosis. The review of Homan et al. [7] deals with neuroimaging and neurophysiological findings and intervention methods such as noninvasive brain stimulation related to this symptom group. Schultz et al. [8] cover important aspects of multimodal neuroimaging approaches combining structural MRI, functional MRI, diffusion tensor imaging (DTI), spectroscopy and positron emission tomography (PET) in order to identify neuronal mechanisms underlying the pathophysiology of schizophrenia and high-risk subjects. Thus, the investigation of the genetic basis of brain pathology is one of the major challenges for future multimodal imaging research.

The described approaches, suitable to improve diagnostic processes and possibly serving as therapeutic targets 
for severe neuropsychiatric diseases, require consolidation on the molecular level. Nothdurfter et al. [9] report on the role of the translocator protein (TSPO) which is involved in neurosteroid synthesis and the role of its neuroprotective ligand XBD173 in the treatment of anxiety and neurological disorders with inflammation-related pathophysiology. Increasing knowledge about disturbed molecular pathways leading to impaired brain function perhaps will allow the development of new pharmacological treatment approaches. As an example, Norman et al. [10] summarize current knowledge on the effects of add-on therapeutic strategies like modafinil, D-cycloserine or oxytocin in severe psychiatric disorder.

Beside pharmacological treatment, psychotherapy is known to alter functional and molecular aspects in distinct brain regions. Specifically, Brakemeier and Frase [11] introduce Interpersonal Psychotherapy (IPT) as an effective short-term treatment strategy in major depression. Schneider [12] portraits the challenging attempt of psychotherapy of suicidal behavior, covering suicide attempts, suicidal intent and ideation. Behavioral therapy aims at modifying behavior, acquisition of techniques for stress reduction and problem-solving strategies.

Regrettably, we here could only illustrate a small selection of the highlights of last year's DGPPN Congress but hope to again provide the participants of our upcoming Congress in 2012 with fruitful insights into new diagnostic and therapeutic developments which someday hopefully will result in improving the life quality of our patients.

\section{References}

1. Gaebel W, Kowitz S, Zielasek J (2012) The DGPPN Research Project on mental healthcare utilization in Germany: inpatient and outpatient treatment of persons with depression by different disciplines. Eur Arch Psychiatry Clin Neurosci. doi:10.1007/ s00406-012-0363-2 (Epub ahead of print)

2. Härter M, Kentgens M, Brandes A, Lambert M (2012) Rationale and Content of psychenet.de- the Hamburg Network for Mental Health. Eur Arch Psychiatry Clin Neurosci. doi:10.1007/s00406012-0359-y (Epub ahead of print)

3. Rössler W (2012) Stress, burnout, and job dissatisfaction in mental health workers. Eur Arch Psychiatry Clin Neurosci. doi: 10.1007/s00406-012-0353-4 (Epub ahead of print)

4. Genius J, Klafki H, Benninghoff J, Esselmann H, Wiltfang J (2012) Current application of neurochemical biomarkers in the prediction and differential diagnosis of Alzheimer's disease and other neurodegenerative dementias. Eur Arch Psychiatry Clin Neurosci. doi:10.1007/s00406-012-0362-3 (Epub ahead of print)

5. Tomasik J, Schwarz E, Guest PC, Bahn S (2012) Blood test for schizophrenia. Eur Arch Psychiatry Clin Neurosci. doi:10.1007/ s00406-012-0354-3 (Epub ahead of print)

6. Ruhrmann S, Klosterkötter J, Bodatsch M, Nikolaides A, Julkowski D, Hilboll D, Schultz-Lutter F (2012) Chances and risks of predicting psychosis. Eur Arch Psychiatry Clin Neurosci. doi: 10.1007/s00406-012-0361-4 (Epub ahead of print)

7. Homan P, Kindler J, Hubl D, Dierks T (2012) Auditory verbal hallucinations: imaging, analysis, and intervention. Eur Arch Psychiatry Clin Neurosci. doi:10.1007/s00406-012-0355-2 (Epub ahead of print)

8. Schultz CC, Fusar-Poli P, Wagner G, Koch K, Schachtzabel C, Gruber O, Sauer H, Schlösser RGM (2012) Multimodal functional and structural imaging investigations in psychosis research. Eur Arch Psychiatry Clin Neurosci. doi:10.1007/s00406-0120360-5 (Epub ahead of print)

9. Nothdurfter C, Baghai TC, Schüle C, Rupprecht R (2012) Translocator protein $(18 \mathrm{kDa})(\mathrm{TSPO})$ as a therapeutic target for neurological and psychiatric disorders. Eur Arch Psychiatry Clin Neurosci. doi:10.1007/s00406-012-0352-5 (Epub ahead of print)

10. Normann C, Nissen C, Frase L (2012) Neuroenhancement strategies for psychiatric disorders-rationale, status-quo and perspectives. Eur Arch Psychiatry Clin Neurosci. doi:10.1007/ s00406-012-0356-1 (Epub ahead of print)

11. Brakemeier E-L, Frase L (2012) Interpersonal Psychotherapy (IPT) in major depressive disorder. Eur Arch Psychiatry Clin Neurosci. doi:10.1007/s00406-012-0357-0 (Epub ahead of print)

12. Schneider B (2012) Behavioural therapy of suicidality. Eur Arch Psychiatry Clin Neurosci. doi:10.1007/s00406-012-0351-6 (Epub ahead of print) 